\title{
Total Hairline Correction in Female Patient
}

\author{
Jae-Heon Jung ${ }^{1}$, In Sik Yun ${ }^{2}$ \\ ${ }^{1}$ Gangnam Yonsei Aesthetic Plastic \\ Surgical Clinic, Hair Transplantation \\ Center, Seoul; ${ }^{2}$ Department of Plastic \& \\ Reconstructive Surgery, Institute for \\ Human Tissue Restoration, Yonsei \\ University College of Medicine, Seoul, \\ Korea
}

No potential conflict of interest relevant to this article was reported.

\begin{abstract}
Background The shape of the hairline and the ratio of the forehead to the face are both important factors for a balanced and attractive face. Because males primarily have a hairline with a rectangular or $M$ shape (a frontotemporal-recessed shape), females with such shapes often have a strong masculine image and appear older. Follicular unit (FU) transplantation was used recently so that the forehead could be reduced naturally and effectively by changing the rectangular or M-shape hairline to a round-shape hairline, thus reducing the forehead. So the author is going to introduce a harmonious, balanced correction, named as total hairline correction that presents not only the frontal midpoint and fronto-temporal line, but also a temporal point, infra-temporal area, and/or sideburn.

Methods Author studied 300 operation patients in last three years whose mean age was 29.3 years (range 19-57 years).

Results The average reduced length was followed: frontal mid-point: $0.63(0-1.5 \mathrm{~cm})$, frontotemporal apex: $3.38(1-4.5 \mathrm{~cm})$, Rt $3.18(1-4.5 \mathrm{~cm})$ Lt, temporal point: 0.91 (0-3 $\mathrm{cm})$ Rt, $0.88(0-3 \mathrm{~cm})$ Lt, infratemporal apex: $0.92(0-1.5 \mathrm{~cm}) \mathrm{Rt}, 0.93(0-2 \mathrm{~cm}) \mathrm{Lt}$.

Conclusions In female patient with $M$ shaped or rectangular hairline, total hairline correction which included not only mid-frontal area and fronto-temporal recession, but also temporal peak, infratemporal area and sideburn is better than simple correction of frontotemporal recess in reducing the facial area and maintaining the aesthetic facial balance.
\end{abstract}

Keywords Total hairline correction, Female hairline, Temporal point, Frontotemporal recess, Sideburn correction

\section{INTRODUCTION}

Both the shape of the hairline and the ratio of the forehead to the face are important factors for a balanced and attractive face [1,2]. Since males primarily have a hairline with a rectangular or $\mathrm{M}$ shape (frontotemporal-recessed shape), if females have such shapes often show a strong masculine image and appear older $[3,4]$.

Received: Oct 2, 2013 Revised: Nov 30, 2013 Accepted: Jan 24, 2014

Correspondence: Jae-Heon Jung Gangnam Yonsei Aesthetic Plastic Surgical Clinic, 101 Teheran-ro, Gangnam-gu, Seoul 135-933, Korea.

E-mail: jjhps@hanmail.net

Copyright @ 2014 The Korean Society for Aesthetic Plastic Surgery.

This is an Open Access article distributed under the terms of the Creative Commons Attribution Non-Commercial License (http://creativecommons.org/licenses/by-nc/3.0/) which permits unrestricted non-commercial use, distribution, and reproduction in any medium, provided the original work is properly cited. www.e-aaps.org
In the past, forehead reduction was performed in a field of plastic surgery, so that the height of the forehead could be reduced, but the effect was negligible due to several reasons such as the scar along the hairline, the inability to correct the frontotemporal recess, and a backstretch phenomenon [5].

However, using the follicular unit (FU) transplantation, the forehead could be reduced naturally and effectively by changing the rectangular- or M-shape hairline to the round shape hairline $[6,7]$.

Specifically, a harmonious and balanced correction is possible by performing a total hairline correction that corrects not only the frontal midpoint and fronto-temporal line, but also a temporal point, infra-temporal area, and sideburn (Fig. 1).

\section{METHODS}

A hairline is defined as the line that forms a boundary of the face 


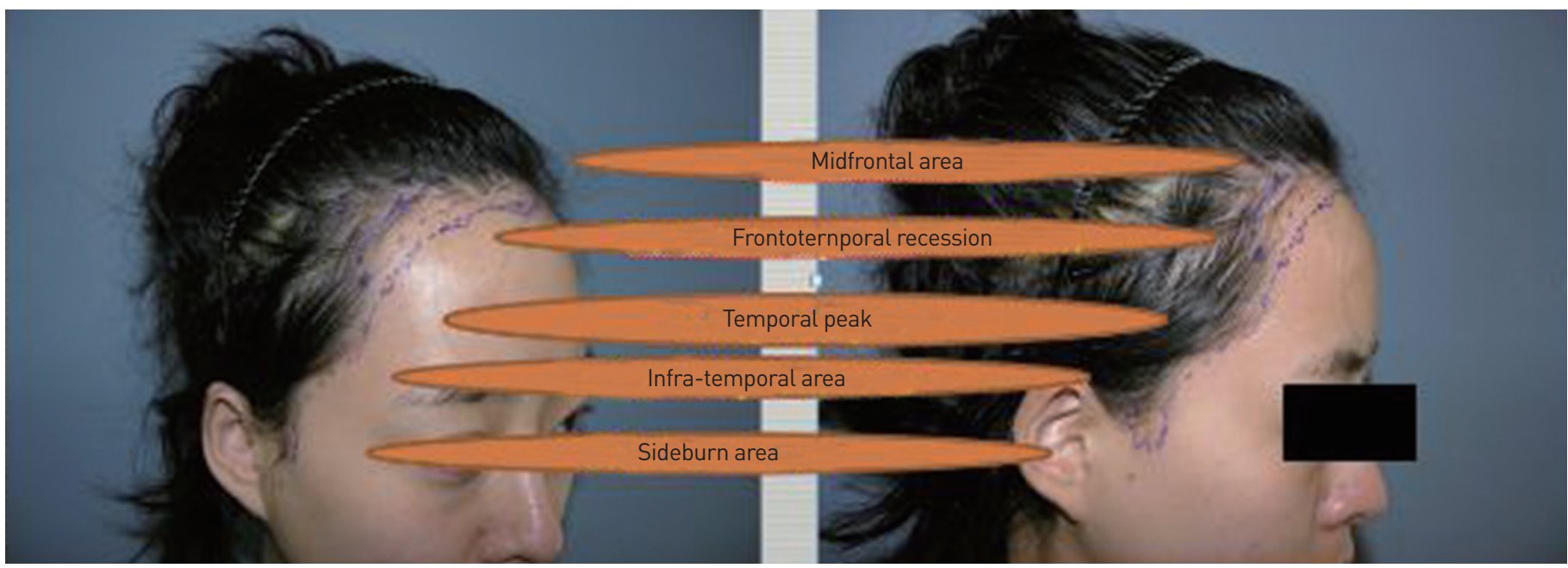

Fig. 1. A hairline is classified by its position. It is divided into a frontal hairline and a temporal hairline based on the fronto-temporal apex as a boundary. The temporal hairline is subdivided into a supra-temporal hairline and an infra-temporal hairline based on a temporal point as a boundary.

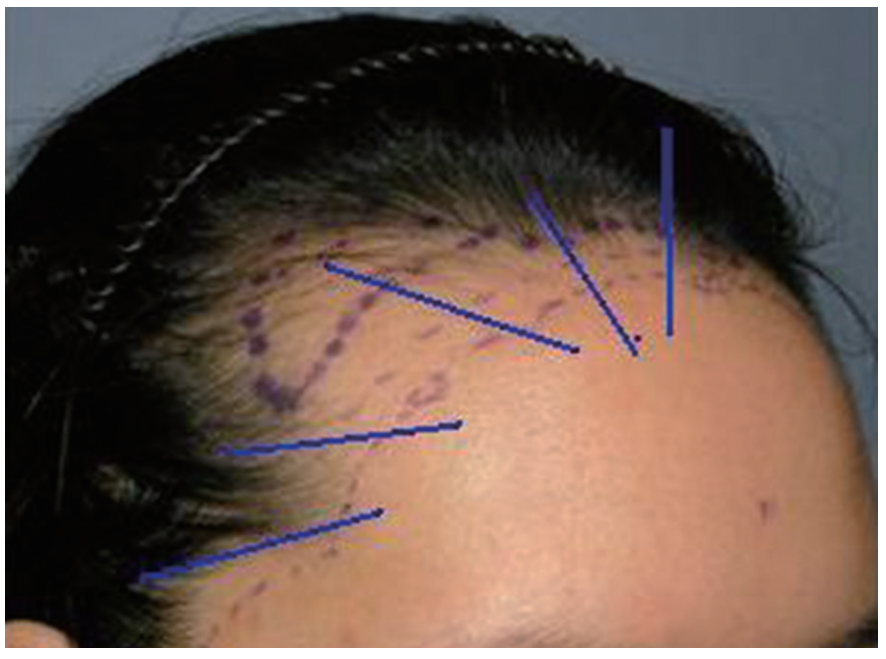

Fig. 2. The position of a part-line is checked and marked, and then the direction of hair around the part-line is identified and marked.

from the scalp and is classified by its position. It is divided into a frontal hairline and a temporal hairline based on the fronto-temporal apex as a boundary.

The temporal hairline is subdivided into a supra-temporal hairline and an infra-temporal hairline based on a temporal point as a boundary.

\section{Surgical procedure}

\section{Preoperative design}

The height of the forehead is often designed slightly higher (5-7 $\mathrm{mm}$ ) than the bottom one-third of the face (from the subnasalis to the menton).

A temporal point should be positioned on a line perpendicular

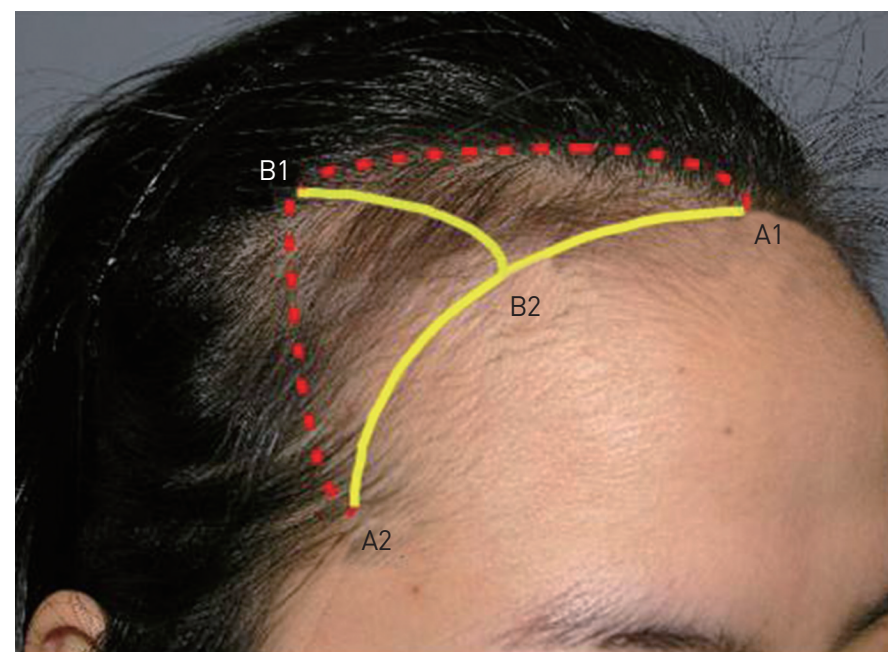

Fig. 3. A line $A$ is defined as the distance from a midfrontal point to a temporal point and line B is defined as the distance from the apical region of the frontotemporal recess to the middle point of line $A$, the area of the triangle is $A \times B / 2$, with $A \times B$ as the approximate area of the recipient since the correction is required on both sides.

to the outer margin of the lateral orbital rim. It is natural to be positioned at least $2 \mathrm{~cm}$ apart from the outer end of the eyebrow.

The position of a part-line is checked and marked, and then the direction of hair around the part-line is identified and marked (Fig. 2).

\section{Measurement of amount to be needed}

To determine how much hair would be required for transplantation, the area of the recipient site must be measured. According to the author's study of 320 patients who had the hairline correction surgery, when line A is defined as the distance from a midfrontal 
point to a temporal point and line B is defined as the distance from the apical region of the frontotemporal recess to the middle point of line $\mathrm{A}$, the area of the triangle is $\mathrm{A} \times \mathrm{B} / 2$, with $\mathrm{A} \times \mathrm{B}$ as the approximate area of the recipient since the correction is required on both sides. The averages of lines A and B in patients who had the surgery were respectively $8.6 \mathrm{~cm}$ and $3.5 \mathrm{~cm}$, and the average correction area was $30.1 \mathrm{~cm}^{2}$ (Fig. 3) [8].

\section{Donor harvesting and preparation}

Local anesthesia is administered by using a tumescent solution, and a single-stripe excision is performed. After trichophytic excision is performed in the inferior edge of the scalp, a hypodermic suture of the 4-0 vicryl and a skin suture of 3-0 nylon are used to close the incision by the continuous interlocking method.

Hair follicles are separated into the FU by using a binocular microscope and sorted into one of four groups, depending on the size, such as thin 1-hair FU, thick 1-hair FU, 2-hair FU, and 3- to 4-hair FU. When a single hair is required, follicular splitting can be performed; on the other hand, when a 3- to 4-hair FU is re- quired, follicular pairing (recombinant technique) can be performed so that the ratio of the FU can be adjusted (Fig. 4).

Since the temporal region or sideburn requires an acute angle on implantation, several methods would be useful to create natural appearance. First, a double-angle technique is performed in which insertion is carried out almost in parallel within the subcutaneous tissue after penetrating the skin at a $20^{\circ}$ to $25^{\circ}$ angle (Fig. 5).

Second, a tip-up technique is performed to direct the hair consistently toward the skin; the hair tip points upward when a follicle is installed after adjusting to the natural hair bend and the hair tip points downward by rotating the hair $180^{\circ}$ and implanting the hair with bevel down.

\section{Postoperative care}

For the donor area, an elastic support bandage was used for 1 day after surgery; on the following day, a dressing procedure was performed for both the recipient and donor areas using shampoo and diluted hydrogen peroxide. After 3rd postoperative days, the use of $3 \%$ minoxidil after shampooing improves wound healing and
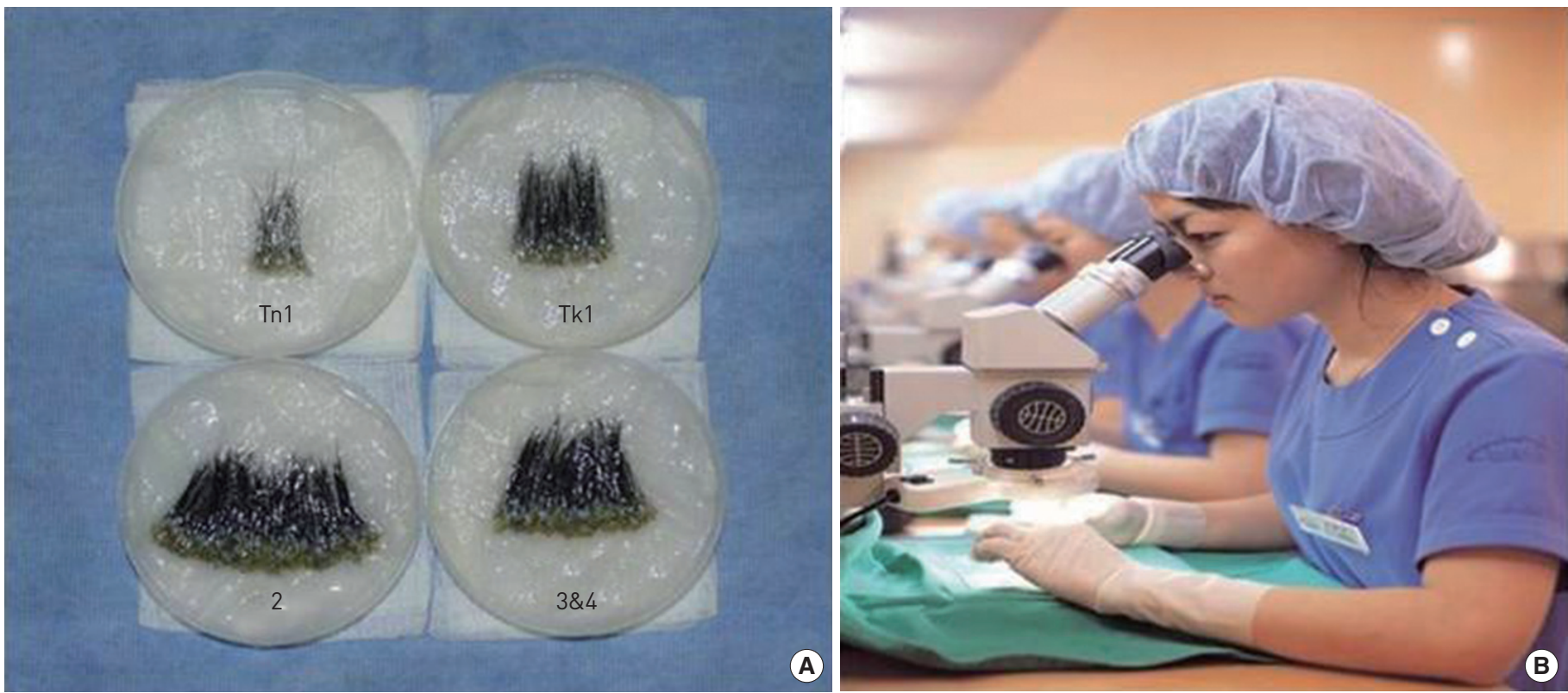

Fig. 4. Each hair follicle is separated from the FU by using a binocular microscope and sorted into one of four groups, depending on the size, such as thin 1-hair FU, thick 1-hair FU, 2-hair FU, and 3- to 4-hair FU.
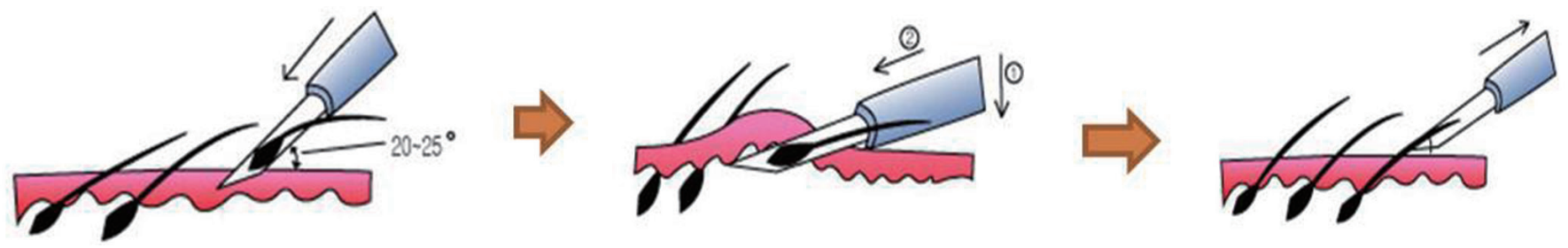

Fig. 5. A double-angle technique is performed in which insertion is carried out almost in parallel within the subcutaneous tissue after penetrating the skin at a $20^{\circ}$ to $25^{\circ}$ angle. 
alleviates the degree of postoperative effluvium that occurs in the early phase of the surgery.

Surgical string was removed approximately 10 days after surgery. Use of color or permanent solutions is recommended to be delayed for 1 month if needed. There is a chance of folliculitis from 1 to 2 months after surgery, although these occur more fre-

Table 1. Advanced length at 4 points

\begin{tabular}{llcc}
\hline Site & & Range & Average length \\
\hline Frontal midpoint & & $0-1.5 \mathrm{~cm}$ & $0.63 \mathrm{~cm}$ \\
Frontotemporal apex & Right & $1-4.5 \mathrm{~cm}$ & $3.38 \mathrm{~cm}$ \\
& Left & $1-4.5 \mathrm{~cm}$ & $3.18 \mathrm{~cm}$ \\
Temporal point & Right & $0-3 \mathrm{~cm}$ & $0.91 \mathrm{~cm}$ \\
& Left & $0-3 \mathrm{~cm}$ & $0.88 \mathrm{~cm}$ \\
Infratemporal apex & Right & $0-1.5 \mathrm{~cm}$ & $0.92 \mathrm{~cm}$ \\
& Left & $0-2 \mathrm{~cm}$ & $0.93 \mathrm{~cm}$ \\
\hline
\end{tabular}
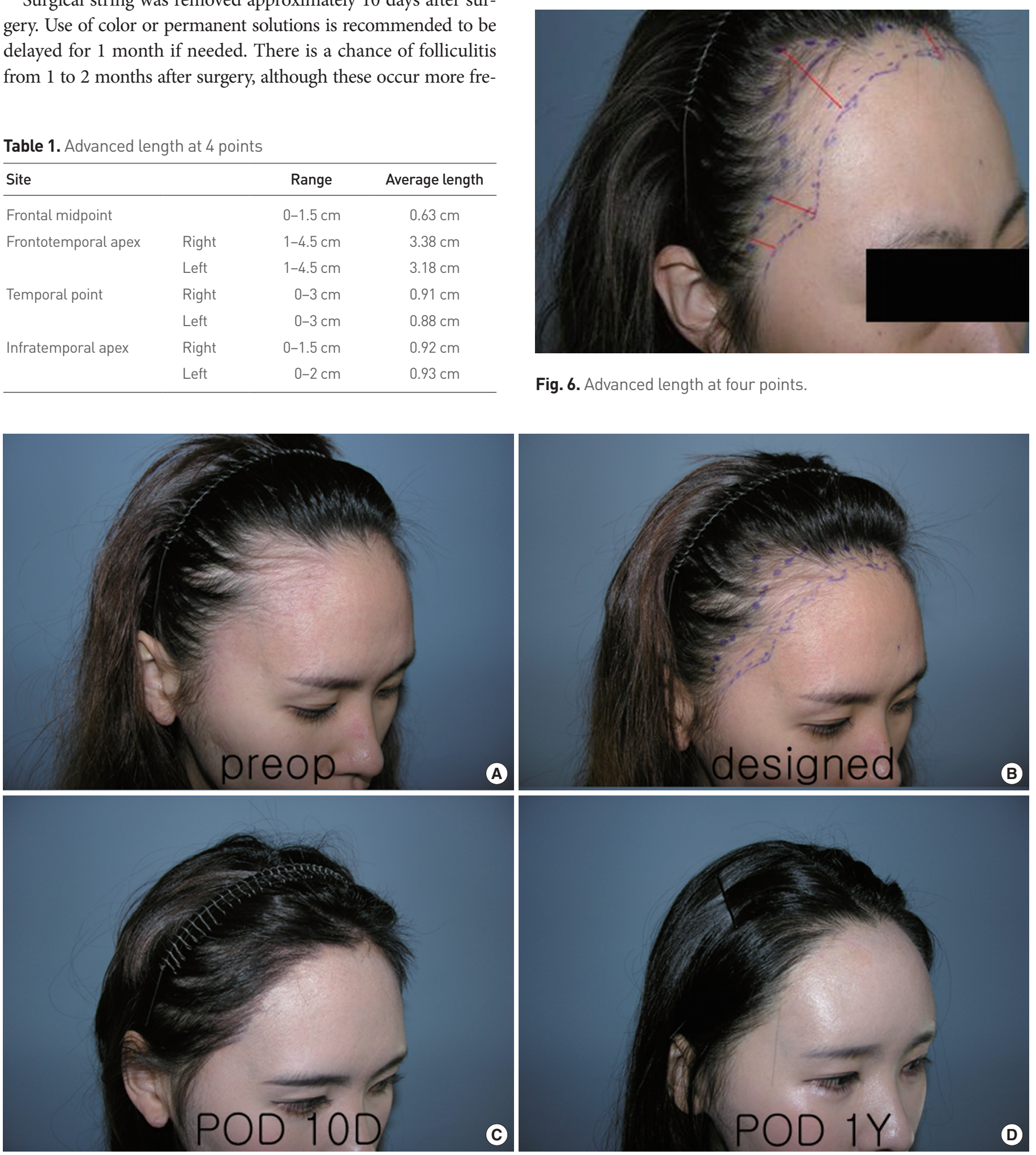

Fig. 6. Advanced length at four points.

quently in patients with oily scalps, but are rare after 3 months.

Fig. 7. The averages of lines $A$ and $B$ in patients who had the surgery were respectively $8.6 \mathrm{~cm}$ and $3.5 \mathrm{~cm}$, and the average correction area was $30.1 \mathrm{~cm}^{2}$. 


\section{RESULTS}

The average patient age (300 patients from 2009 to 2011) is 29.3 years old-range from 19 to 57 years old. The averages of lines A and $B$ in patients who had the surgery were respectively $8.6 \mathrm{~cm}$ and $3.5 \mathrm{~cm}$, and the average correction area was $30.1 \mathrm{~cm}^{2}$ (Table 1 , Fig. 6, 7).

\section{DISCUSSION}

An ideal ratio is considered when the height of the forehead is one-third of the overall length of the face (from a midfrontal point to the menton) [2]. However, 1:1:0.9 is the preferred ratio recently that allows a younger-looking appearance; thus, the height of the forehead is often designed slightly higher $(5-7 \mathrm{~mm})$ than the bottom one-third of the face (from the subnasalis to the menton).

According to the author's study (2007), the hairlines of Asian females can be generally classified into one of the following five types (Fig. 2) [9]. Round (27\%), M-shape (28\%), and rectangular (27\%) foreheads account for the majority of shapes; these percentages are similar to what Dr. Nusbaum reported regarding forehead shapes for women in the West, including $61 \%$ who had a concave,

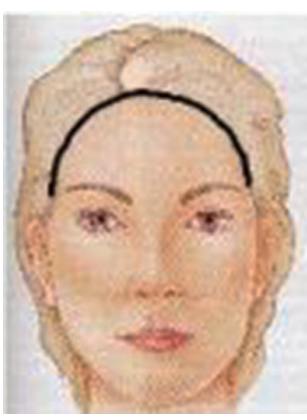

Round type $27 \%$

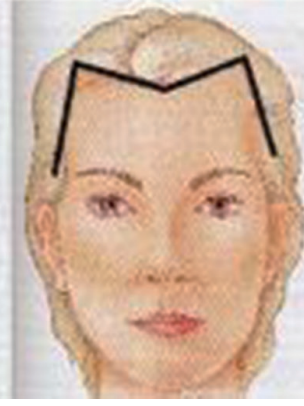

M shape 28\%

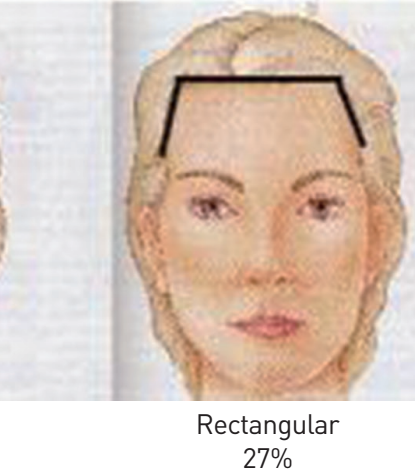

In 234 Volunteer women (2007)

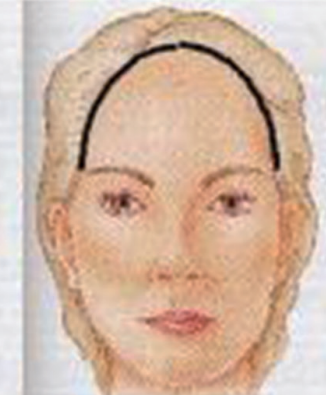

Bell shape $10 \%$

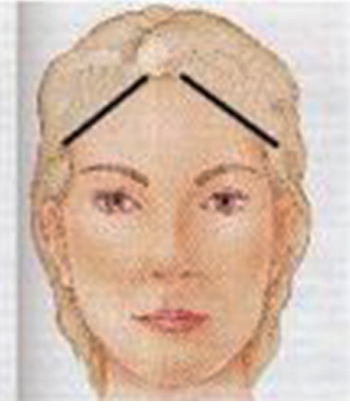

Triangular $3 \%$

Fig. 8. Round type: The frontotemporal recess is not present; the hairline possesses a concave shape and a representative forehead shape that is commonly seen in women. M-shape type: The frontotemporal recess is severe and the lateral end of the frontal hairline is positioned upward in a lateral view and the fronto-temporal angle is less than a $90^{\circ}$ in general. Rectangular type: The frontal line is horizontally positioned, the frontotemporal recess is not severe, and the frontotemporal angle is $90^{\circ}$ or larger. Bell type: The width of the forehead is within the normal range, but the height of the frontal midpoint is $2 \mathrm{~cm}$ higher than average. Triangular type: The hairline moves down nearly straight from the center of the forehead to the temple, and there are numbers of vellus hairs.
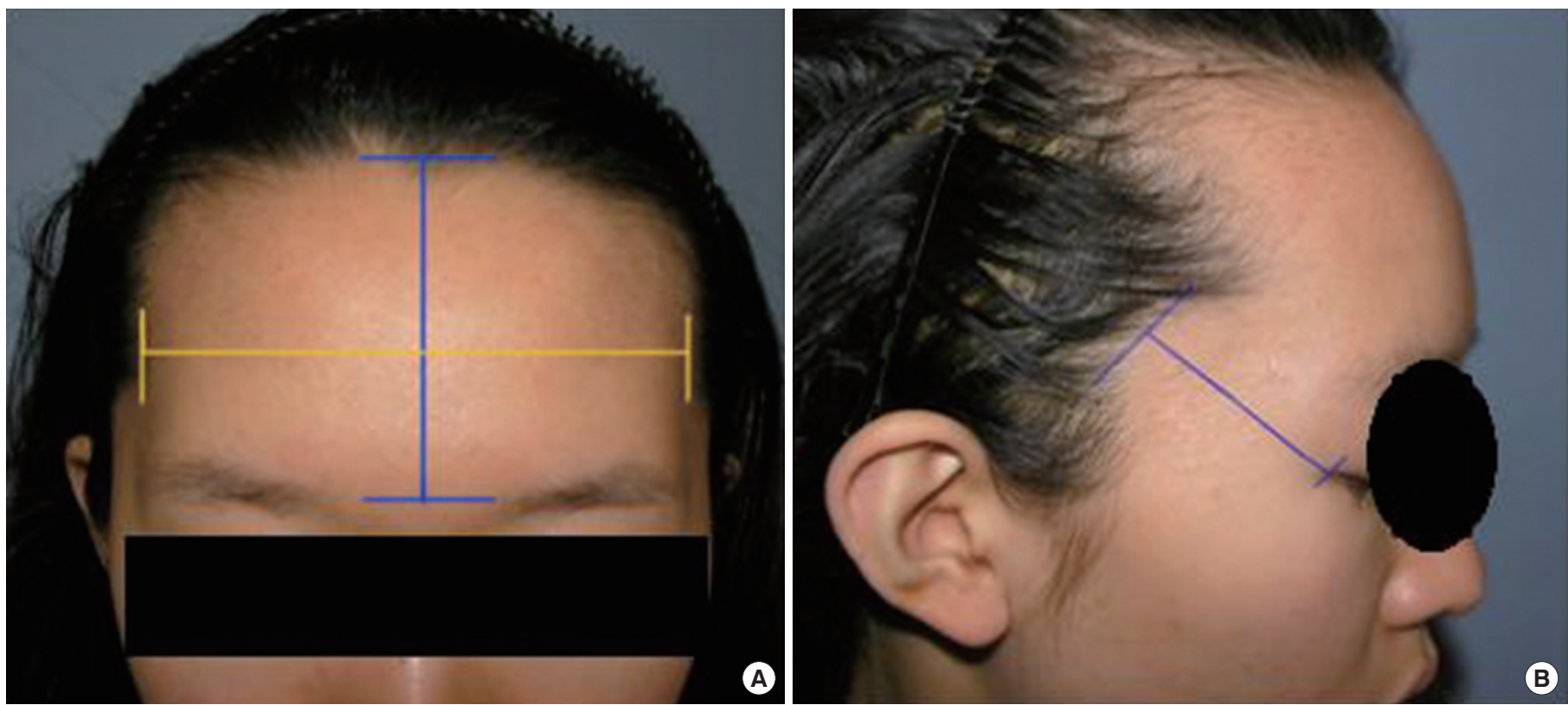

Fig. 9. The measurement of foreheads for Korean women. 
triangular type (corresponding to the M-shape and rectangular foreheads) and $26 \%$ who had a concave and oval type (corresponding to the round forehead, Fig. 8) [10].

The average measurement of foreheads for Korean women reported by the author is as follows (Fig. 9):

- Average height of forehead (mid-eyebrow to base of widow's peak): $6.38 \mathrm{~cm}$

- Average width of forehead (distance between both temporal points): $14.3 \mathrm{~cm}$

- Average depth of the infratemporal recess (infratemporal apex to lateral canthus): $4.29 \mathrm{~cm}$

Nusbaum et al. reported that a widow's peak was observed in $81 \%$ of patients, a lateral peak was present in the midportion between the frontal midpoint and a temporal point, and a temporal point was positioned $1 \mathrm{~cm}$ away from a line drawn perpendicular to the lateral canthus [10].

To correct a hairline, a part-line is one of the important factors that provide a natural appearance. It was observed that $46 \%$ of people had a part-line on the left, $14 \%$ had a part-line on the right, $20 \%$ had a part-line in the middle, and $20 \%$ had part-lines on both sides. In cases of an M-shape forehead, a part-line was not clearly visible, since a conventional partline is positioned toward the inside of the frontotemporal recess. After a surgery, a new part-line is formed in a forward location and is thereby clearly visible. The hair on the most-frontal area of the part-line is directed in a radial pattern, so that the scalp is easily more exposed. For this reason, the density of hair around appears to be relatively low after surgery.

Therefore, if a part-line is positioned within the center onethird of the frontal hairline, it would be natural not to correct width more than $1 \mathrm{~cm}$ as a new frontal midpoint. If a part-line is positioned at the outer one-third of the frontal hairline, a frontal midpoint could be moved down by $1.5 \mathrm{~cm}$, since the center would be camouflaged well by laterally directed hair.

In cases that part-lines are on both sides, strong part-lines are maintained. If one of the part-lines were weak, a more natural hairline would be visible as the part-line on the stronger side.

In cases that the donor hair has a thick caliber, the volume of the hair in the recipient looks natural initially after transplantation, but it does not look natural later because the thick hair will grow in the transition zone. In this case I try to increase the proportion of 1 hair FU, especially thin 1 hair FUs which were disposed on the most frontal area of hairline zone.

If the caliber of donor hair is thin, a transition zone looks more natural, but the volume of the mid- and apex zones on the backside is decreased, so that it is better to increase the hair density on these zones with 3 \& 4 hair FUs.

In cases that the existing frontal hairline zone looks see-through due to innate low-hair density or Hamilton-type female pattern hair loss, enhanced density should be required and can be achieved by adding 2 hairs FU sat into a width of approximately $1-1.5 \mathrm{~cm}$.

A cowlick is a type of part-line which the hair points naturally upward, as seen in $64 \%$ of women in the West. It is more common with a cowlick on the left.

When performing hair transplantations in this area, since the hair directs upward, the shivering effect is decreased and the density appears to be decreased. Therefore, It is better to increase the density of 1 hair FU more than $50 / \mathrm{cm}^{2}$ and reduce the height of corrected area within $1 \mathrm{~cm}$ if possible.

When the forehead is wide, the side of the face appears to be wide due to wide infratemporal area and the posterior location of a temporal point. In general, the shape of the infratemporal line is natural when it is concave in accordance with the curvature of the orbital rim, but it can be designed to a nearly straight line to reduce the infratemporal area or sideface.

When a sideburn is well developed, it can be connected naturally to an infratemporal line. However, when there is no sideburn, sideburn transplantation can be performed at the same time, since the side of the face would look wide without the sideburn.

Unlike men's sideburns, such hair transplantation should be limited to the level of the zygomatic arch and directed posteriorly and inferiorly. Additionally, it is desirable to make the density below the $20 / \mathrm{cm}^{2}$ and avoid using coarse hairs during the transplantation.

After surgery, the side of the face can be partially hidden or made to look smaller by growing the hair as it is; alternatively, the hair can be swept behind the ears and styled naturally.

For a newly designed forehead height, a new midfrontal point should be determined from the mid-eyebrow at a distance of approximately 5 to $7 \mathrm{~mm}$ longer than the lower one-third of the face (subnasalis to menton); the correct width should not exceed 1 to $1.5 \mathrm{~cm}$ from the previous mid-frontal point.

From the new mid-frontal point, the hairline begins to draw laterally in a round shape considering the contour of the face and meet the temporal point that is on the line perpendicular to the lateral orbital rim. An infratemporal line should be designed as a round shape in accordance with the curvature of an orbital rim from the temporal point and then connected to the anterior border of the sideburn. The back edge of the transplanted area should be marked with gentian violet to classify the range of the frontotemporal triangle into three zones as follows: 1) a transition zone, which is positioned at the front and has an approximate width of $1 \mathrm{~cm} ; 2$ ) a mid-zone, which is positioned in the middle and has a width of 1.5 to $2 \mathrm{~cm}$ and 3) an apex zone, which is positioned in the apical region and has a triangular shape.

To prevent a straight hairline, a zigzag or a snail-tract shape is made, and 8 to 10 clusters, including a widow's peak and lateral peak, should be designed to avoid symmetrical appearance of 


\section{both sides.}

If the transplantation density was 70 hairs $/ \mathrm{cm}^{2}, 2,107$ hairs would be required. However, the total hair required for the transplantation should be estimated after considering the additional hair needed for transplantation in the infratemporal and sideburn areas.

The density of the occipital region is calculated by using trichoscopy or densitometry; then, the size of the needed strip is measured by determined length and a width within the ranges of 20 to $22 \mathrm{~cm}$ and 0.8 to 1.5 in most patients, respectively.

When the thickness of the patient's hair is in the average range (60 to 80 microns), the ratio of 1-hair FU:2-hair FU:3- to 4-hair FU should be 3:4:3, but when the patient has thick hair ( $>80 \mathrm{mi}-$ crons), the transition zone should be deliberately widened by increasing the ratio of the 1 -hair FU by $50 \%$ to have a natural-looking transition zone. In contrast, in cases of thin hair ( $<60$ microns), the mid-zone should be widened by increasing the ratio of the 2hair FU artificially to increase the volume of the hair.

Each FU changes depending on the transplanted region. Only 1-hair FU is transplanted to have a natural-looking transition zone and make a dense packing. In the mid-zone, a 2-hair FU is transplanted, whereas 3- to 4-hair FU is primarily transplanted in the apex zone to increase the volume. However, when the density of the FU is decreased in the mid-or apex zone, it can be increased by transplanting a 1-hair FU between them (Fig. 10).

In the transition zone, the dense packing is needed so that we make a density at 50 to $60 / \mathrm{cm}^{2}$ with 1 -hair FU. The mid- and apex zones are transplanted at densities of 30 to $40 / \mathrm{cm}^{2}$ with 2-hair FUs and 20 to $25 / \mathrm{cm}^{2}$ with 3 to 4 -hair FUs.

The direction of hair will be most natural to follow the existing

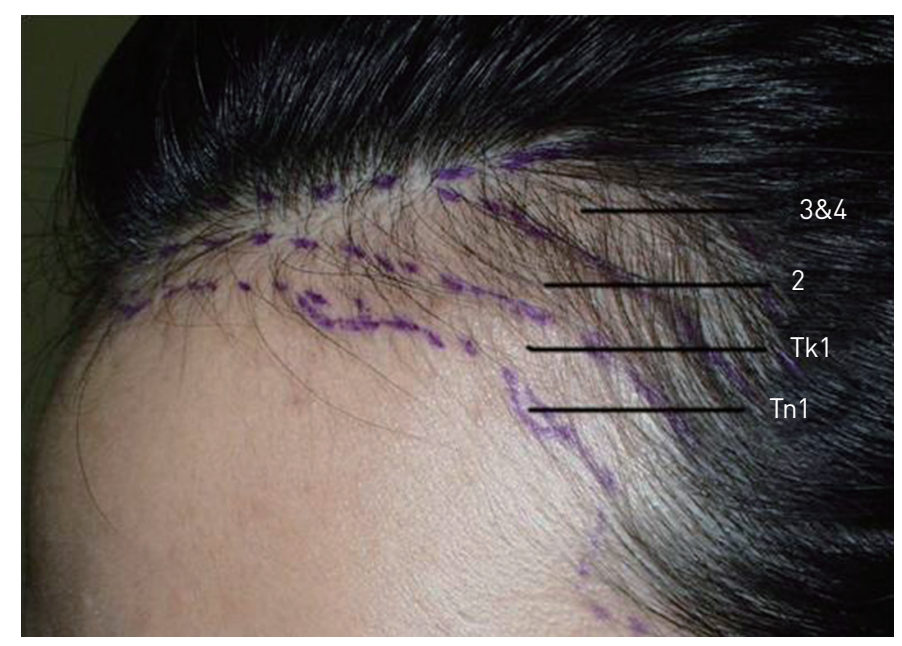

Fig. 10. Each FU changes depending on the transplanted region. Only 1-hair FU is transplanted to have a natural-looking transition zone and make a dense packing. In the mid-zone, a 2-hair FU is transplanted, whereas a 3- to 4-hair FU is primarily transplanted in the apex zone to increase the volume. hair line along the frontal hairline. In general, temple will show patterns that lower portion will be strongly from inferoposterior direction toward laterally changed to lateral direction, then as approaching to part-line it will again be facing to downward.

The angle of hair will get larger from sideburn toward part-line. It is about 15 to 20 degree up to temporal point, about 20 to $30^{\prime}$ up to supratemporal line, about 30 to $45^{\prime}$ around lateral frontal line, and about 45 to $60^{\prime}$ around central frontal line. It'll be natural to transplant at 60 to $90^{\prime}$ near to the part-line area.

In Asian patients, a few cases of severe curly hair over the recipient sites were observed. However, they were improved after 1 or 2 years.

In transgender patients (male-to-female transgender patients), as male pattern hair loss progresses, there is a chance of hair loss at the rear of the transplantation site. It is, therefore, necessary to minimize the range of correction required, considering secondary transplantation via a conservative design and confirming sufficient hair in the occipital region if necessary.

If there are scars due to injury, burns, and surgeries, such as forehead reduction or facial lifting, hair transplantation can be done on the scar sites as well; given the lower survival rate of hair in these areas, it is helpful to use the 'no touch technique, which uses a hair transplanting device after making slits for better graft survivals.

Laser hair removal treatment may produce non-natural straight hairline for several reasons: 1) There is no 1-hair FU located at the front of the hairline, and the hairline looks too dense with thick 2-hair FUs are exposed in front; 2) There is a distinctively clear and sharp straight hairline without either a widow's peak or lateral peaks or 3) A white band of depigmentation appears at the skin border of the hairline.

In most cases, natural hairlines can be recreated by making multiple peaks and clusters and irregular zigzag lines with single hairs on the frontal hairline and correcting the width of the forehead.

\section{REFERENCES}

1. Holcomb JD, McCollough EG. Trichophytic incisional approaches to upper facial rejuvenation. Arch Facial Plast Surg 2001;3:48-53.

2. Ramirez AL, Ende KH, Kabaker SS. Correction of the High Female Hairline. Arch Facial Plast Surg 2009;11:84-90.

3. Shapiro R. Principles and techniques used to create a natural hairline in surgical hair restoration. Facial Plastic Surgery Clinics of North America 2004;12:201-17.

4. Beehner M. Hairline Design in Hair Replacement Surgery. Facial plast Surg 2008;24:389-403.

5. International Society of Hair Restoration Surgery: 2007 practice census results. Chicago: RH Research; 2007.

6. Lee TS, Minton TJ. An update on hair restoration therapy. Current 
Opinion in Otolaryngology \& Head \& Neck Surgery 2009;17:287-94.

7. Rawnsley JD. Hair Restoration. Facial Plastic Surgery Clinics of North America 2008;16:289-97.

8. Farjo B. Estimating graft numbers made eay for the recipient site. H T Forum Int 2001;11:101.
9. Jung JH, Rah DK, Yun IS. Classification of the female hairline and refined hairline correction techniques for Asian women. Dermatol Surg 2011;37:495-500.

10. Nusbaum BP, Fuentefria $S$. Naturally occurring female hairline patterns. Dermatologic Surgery 2009;35:907-13. 